\title{
WMR Navigation Using Local Potential Field Corridors and Narrow Local Occupancy Grid Perception
}

\author{
Lluís Pacheco, Xavier Cufí, Ningsu Luo and Javier Cobos \\ Institute of Informatics and Applications \\ University of Girona, Spain \\ \{lluispa,ningsu,xcuf\}@eia.udg.es
}

\begin{abstract}
Path planning and control strategies applied to autonomous mobile robots should fulfil safety rules as well as achieve final goals. Trajectory planning applications should be fast and flexible to allow real time implementations as well as environment interactions. The methodology presented uses the on robot information as the meaningful data necessary to plan a narrow passage by using a corridor based on attraction potential fields that approaches the mobile robot to the final desired configuration. It employs local and dense occupancy grid perception to avoid collisions. The key goals of this research project are computational simplicity as well as the possibility of integrating this method with other methods reported by the research community. Another important aspect of this work consist in testing the proposed method by using a mobile robot with a perception system composed of a monocular camera and odometers placed on the two wheels of the differential driven motion system. Hence, visual data are used as a local horizon of perception in which trajectories without collisions are computed by satisfying final goal approaches and safety criteria.
\end{abstract}

Index Terms - mobile robotics, local path planning, robot vision control.

\section{INTRODUCTION}

Robot trajectory planning is a challenge currently faced by robotic researchers. Preliminary work over the last decades has focused on obtaining the configuration space (C-space) that allows a collision free path to be computed [1]. In this context, the allowed navigation control signals should fulfil obstacle avoidance policies as well as achieve the final desired coordinates. The scientific community has carried out several studies in this field. Some approaches use artificial potential fields which attract mobile robot to the desired objective and create repulsive forces to achieve obstacle avoidance [2-3]. The main drawback of potential fields is local minimal failures. However, their flexibility is reported as an advantage when small or moving obstacles are met.

Methods that do not suffer from local minimal failures, known as Probabilistic Roadmap Methods (PRMs), have been developed [4-5]. They consist of two phases: in the first, nodes are generated considering the different robot configurations from the $\mathrm{C}$-space and the connectivity of $\mathrm{C}$ space nodes is obtained, and in the second node connections of pairs of nearby configurations are selected as candidates to be tested by local planners. Thus, the pairs of connected nodes are stored as roadmap edges. The start and goal can be connected by a graph, and the path is obtained by running shortest path search algorithms such as Dijkstra's. The drawbacks of PRM are the generation of unattractive paths and a lack of flexibility. Path optimization can be improved using local motions controlled by local potential fields [6-7]. Other topics of research are the importance of the local information and knowledge of vehicle dynamics when avoiding obstacles as well as the policies for achieving the final desired coordinates, known as the dynamic window approach [8]. It allows reactive obstacle avoidance with limited acceleration and velocity derived from robot motion dynamics. Hence, some approaches to mobile robots propose the use of potential fields that satisfy the stability in a Lyapunov sense with a short prediction horizon, as proposed in [9]. Furthermore, the occupancy grid provides a robust and unified approach to a variety of problems in spatial robot perception and navigation [10]. The occupancy field can be depicted by a probability density function that relates sensor measures to the real cell state. The Bayesian estimation formulation has been proposed by researchers for static occupancy, not only for the use of the last sensor observation measure. Thus, the last occupancy estimate should also be considered. Using 2D grids for static indoor mapping is proposed in [11]. Other works propose multidimensional grids in which multi target tracking algorithms with obstacle state space are used with Bayesian filtering techniques [12]. Integrating perception and planning is an interesting topic. For example, planning cycles that consider a time horizon in which partial trajectories are planned until the robot state is close to the goal are reported in [13]. Another approach [14] uses a narrow field of view to reach a final desired robot configuration through a local dense perception grid provided by the on robot sensors, in which only the significant obstacle vertex points are considered.

The present research can be understood as a fast, flexible methodology. Therefore, a local objective is reached by using local perception knowledge. In this sense, global, high-level planners can fulfil intermediate local objectives improving the system presented when local minimal failures occur. Another important idea consists of facing the robot towards the desired objective. Hence, control is understood as a sequence of steering actions followed by a straight line tracking command. Moreover the idea presented is implemented by using the available lab robot platform, which consists of a differentially driven mobile robot with a free rotating wheel that uses a simple perception system consisting of a monocular camera and an odometer system. Hence, a narrow occupancy grid as well as positions and velocities can be obtained.

This work introduces an extension of the path planning methodology reported in [14] and the paper is organized as 
follows. In Section I, the main ideas and research objectives are presented. Section II presents the methodology developed from a general point of view, as well as the cost function and algorithms used to obtain the local desired cell considering the significant obstacle magnitudes depicted by the local grid perception. Section III applies the concepts introduced in Section II in a practical approach considering the data provided by a monocular camera as well as the robot coordinate information that comes from the robot dead reckoning system based on incremental encoders. Finally, in Section IV the concerning drawbacks and successes within a wide scope are analysed and some conclusions are presented. Future research trends are also reported.

\section{CORRIDOR TRAJECTORY PLANNING WITHIN A LOCAL NARROW OCCUPANCY GRID PERCEPTION HORIZON}

Corridor planning for natural agents has been presented as a new and useful robot control and planning framework using low level obstacle avoidance and simple control [15]. Butler has developed a system in which innate local navigation animal abilities are combined with a very simple imposed path-like structure to produce a desired overall motion. [7] proposes using previously computed corridors within free $\mathrm{C}$-space to guide the global motions of the robot while local motions are led by an attraction point that is moving along the backbone path of the corridor. This work proposes that local motions can be generated considering a single attraction point. Obstacle avoidance is fulfilled by only considering the local perception information and free Cspace is used to plan feasible trajectories that approach the robot to the goal. In this section we introduce the potential field corridor concepts considering that an occupancy grid is provided by an on robot perception system. The algorithm is described and general conclusions of the overall method are also briefly presented.

\section{A. Corridor Potential Field Formulation}

This work pertains to particular indoor environments with flat floor surfaces; however, it can also be applied to outdoor environments. The occupancy grid is a well known environment description technique, which is useful in a variety of robotic tasks [10]. Range finders such as laser or sonar beams can build an occupancy grid as a discrete stochastic process defined over a set of continuous spatial coordinates $(x, y)$. Hence, the space is divided into a finite number of cells representing a $2 \mathrm{D}$ position, $1 \leq j \leq R \quad 1 \leq i \leq C$. The $R$ and $C$ parameters are the number of rows and columns of the grid respectively. The cell column coordinates are designated by $X_{i}$ and the rows by $Y_{j}$. It is assumed that local occupancy grid data is provided by the on robot perception system. The occupancy probability is divided into only two ranges: free and occupied. The grid can be updated by using the sensor models and the current information. Hence, given a sensor measurement $m$, the occupancy probability $P\left(O_{X i Y_{j}}\right)$ for the different cells can be computed by applying Bayes rule, $P\left(O_{X i Y j} / m\right)=$ $P\left(O_{X i Y_{j}}\right) P\left(m / O_{X i Y j}\right)$. Hence, the probability that a cell is occupied $P\left(O_{X Y Y j} / m\right)$ is given by the cell sensor measurement $P\left(m / O_{X i Y j}\right)$, and the previous occupancy cell state $P\left(O_{X i Y j}\right)$. The Bayesian approach has led to the research methodology reporting aspects such as sensor fusion, multidimensional grids used to deal with mobile obstacles, and probabilistic velocity obstacle approach studies [12, 16 and 17]. However, in this section it is considered that the occupancy probability for each cell is given; hence, the free cells have less probability of being occupied than a threshold $t_{l}$ has. Once the free occupancy cells are known, the problem is formulated as finding the optimal cell that brings the WMR (wheeled mobile robot) close to the desired coordinates $\left(X_{d}\right.$, $\left.Y_{d}\right)$ by finding the closest local desired coordinates $\left(X_{l d}, Y_{l d}\right)$ within the local perception grid $\left(X_{i}, Y_{j}\right)$. In this sense, perception is considered as a local receding horizon in which the trajectory is planned. The local desired cell is obtained by minimizing a cost function $J$ that should act as a potential field corridor. Thus, the cost function is minimized by attracting the robot to the desired objective through the free available local cell coordinates. Due to the narrow field of perception, the robot should be oriented towards the goal. Hence, the $\theta_{l g}$ value depicts the maximal angle that can be attained within the local grid. Thus, first, the orientation error towards the goal should be minimized:

$$
\begin{aligned}
& \text { When } \operatorname{atan}\left(Y_{d} / X_{d}\right)=\theta_{d}>\theta_{\mathrm{lg}} \\
& \quad J(i, j)=\min \left(a b s\left(a \tan \left(Y_{j} / X_{i}\right)-a \tan \left(Y_{d} / X_{d}\right)\right)\right) \\
& \forall 1 \leq i \leq \mathrm{R}, 1 \leq j \leq C \Leftrightarrow P\left(O_{X_{i} Y_{j}}\right)<t_{1}
\end{aligned}
$$

Once the WMR is oriented towards the goal, two cost functions are proposed as potential fields: the Euclidean distance and the corresponding one-dimensional differences:

$$
\begin{aligned}
& \text { When } \operatorname{atan}\left(Y_{d} / X_{d}\right)=\theta_{d} \leq \theta_{\mathrm{lg}} \\
& \text { A) } J(i, j)=\min \left(\left(X_{i j}-X_{d}\right)^{2}+\left(Y_{j}-Y_{d}\right)^{2}\right)^{1 / 2} \\
& \text { B) } J(i, j)=\min \left(a b s\left(X_{i j}-X_{d}\right)+a b s\left(Y_{j}-Y_{d}\right)\right) \\
& \forall 1 \leq i \leq \mathrm{R}, 1 \leq j \leq C \Leftrightarrow P\left(O_{X_{i j} Y_{j}}\right)<t_{1}
\end{aligned}
$$

Fig. 1 depicts the above minimization problems that arise from the narrow field of view perception. The constraints considered are:

$$
\left\{\begin{array}{l}
\left(U_{R}, \Delta U_{R}, U_{L}, \Delta U_{L}\right) \leq\left(C_{R}, C_{\Delta R}, C_{L}, C_{\Delta L}\right) \\
\left|x_{l d}, y_{l d}\right|-\left|x_{o}-y_{o}\right| \geq C_{o} \\
|X(k+1), Y(k+1)|-\left|X_{d}, Y_{d}\right| \leq \alpha|X(k), Y(k)|-\left|X_{d}, Y_{d}\right| \\
\alpha \in[0,1)
\end{array}\right\}
$$

Parameter $k$ is used for referencing the instant of time. The limitation of the input signal is taken into account in the first constraint as a function of the local desired points and the WMR dynamics. Thus, $U_{R}$ and $U_{L}$ are the inputs, $C_{R}$ and $C_{L}$ are the referred constraints, and $C_{\Delta R}$ and $C_{\Delta L}$ are the corresponding increment bounds. The second constraint, $C_{o}$, is related to the obstacle points, $\left(X_{o}, Y_{o}\right)$, and should include a $W P$ heuristic (wide-path) related to the WMR dynamics [1]. The wide-path is a heuristic concept, and should be enough to avoid robot collision. Fig. 1 shows these concepts. The safety distance is obtained by:

$$
\left\|V_{1}-A\right\|>W P / \sin \left(\pi / 2-\theta_{l d}\right)
$$

where the significant obstacle vertex is represented by $V_{l}$ and $\left\|V_{1}-A\right\|$ denotes the Euclidean distance between $V_{l}$ and $A, \theta_{l d}=\operatorname{atan}\left(Y_{l d} / X_{l d}\right)$. The last constraint, represented in (3), is only a convergence criterion by selecting $\alpha$ less than unity. 
The algorithm explores the grid only considering the free positions. When no obstacle is met by using (1) and (2-a) or (2-b), the minimal distance corresponds to the optimal local coordinates, $\left(X_{l d}, Y_{l d}\right)$, that should be reached. However, when obstacles are found, the maximal obstacle vertex that comes closest to the goal is considered as the local objective, and acts as a local potential field. Hence, considering $G_{1}$ as the goal and the local grids acquired in frames $f 1$ and $f 2$, as shown in Fig. 1, a path planning trajectory is given by following the straight lines $S A, A A^{\prime}$, $A^{\prime} G_{l}$. Each new line can be followed by commanding a steering action followed by a straight line. In this sense the WP should consider these aspects. When elastic band theory is considered the steering action can be commanded in the areas with bigger safety distances [18]. Hence, the WP necessary along a narrow path is reduced. In the present work a narrow field and feasible perception system are assumed. Therefore, the steering action is limited, and the trajectory is planned by acquiring multiple approaching grid frames, as shown in Fig. 2. Hence, the $S A$ trajectory is considered in $f_{l}$, then in $f_{2}$ the desired point is moved backwards to $A$ ', and in $f_{3}$ it is moved forward to A', This results in the potential field being smoothly oriented, which minimizes the WPs necessary. Moreover, the significant vertex, as for example at the start $\left(S, V_{l}, V_{2}\right)$, can be joined by a triangle. The following path planning grids are also consecutive triangles containing the robot initial configuration that can be connected. In this way continuous field vectors can be obtained [19].

\section{B. The Local Grid Path Finding Algorithms}

The algorithm should find the local cell that best approaches the goal using the local grid provided by the perception system, where the occupied cells are known. When no obstacle is found the procedure consists of selecting the optimal approaching cell.

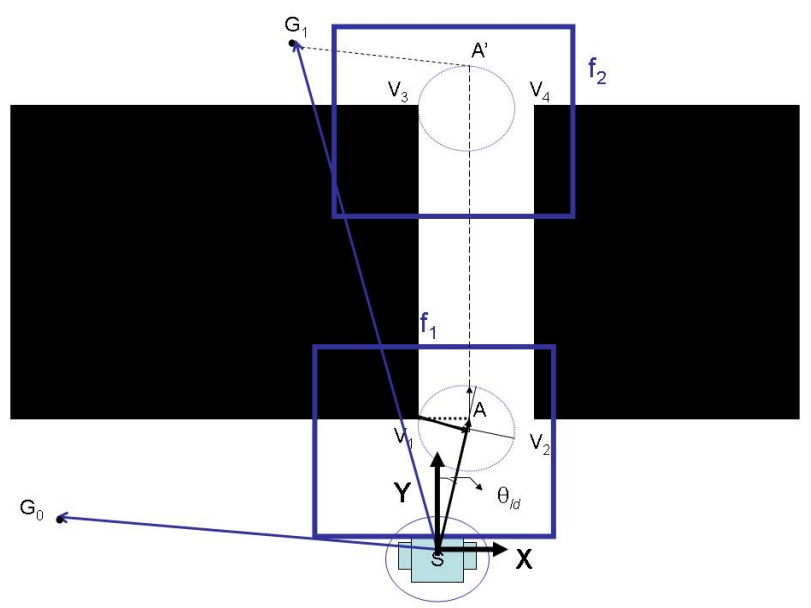

Fig. 1: Two different desired points, $\mathrm{G}_{0}$ and $\mathrm{G}_{1}$, produce different cost function optimizations. In the $\mathrm{G}_{0}$ case, the local desired coordinates are computed by first minimizing equation (1). However, in the case of $\mathrm{G}_{1}$, (2-a) or (2-b) can be used.

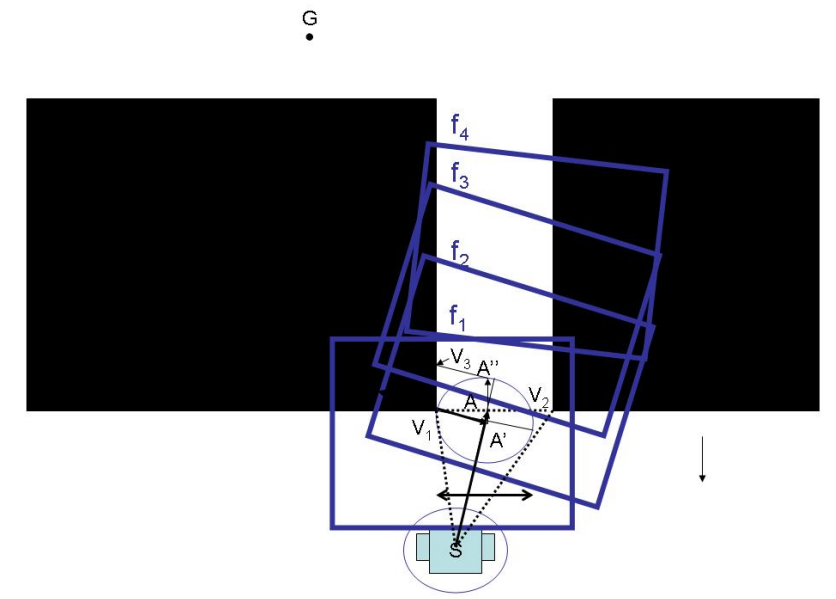

Fig. 2: Using efficient perception systems result in smoother steering actions as a consequence of smoother potential fields.

When obstacles are met, their biggest left or right vertex dimensions are considered. In this context, the perception algorithms compute the number of visible obstacles as well as the largest dimension grid coordinates (up, down, left and right). The vertex that comes closest to the objective, where the mobile robot path is possible, is selected as the meaningful vertex. Fig. 3 provides the algorithm pseudo code that gives the meaningful vertex. Once the vertex is obtained, it should be verified that the path between the closest obstacles is possible. Fig. 4 illustrates the procedure using pseudo code.

The algorithm depicted in Fig. 4 looks for a possible path between columns or rows. The path between rows is possible when an obstacle is partially occluding another obstacle located behind it.

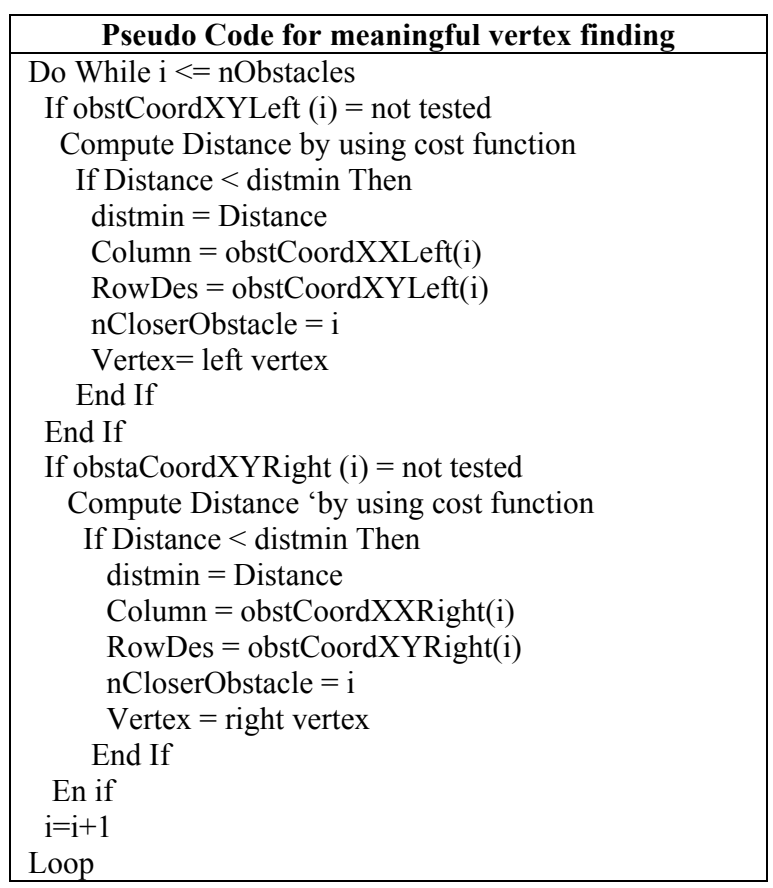

Fig. 3: Pseudo-code search for the meaningful vertex coordinates. The vertex obstacle position, right or left, and number of obstacles are also obtained. 


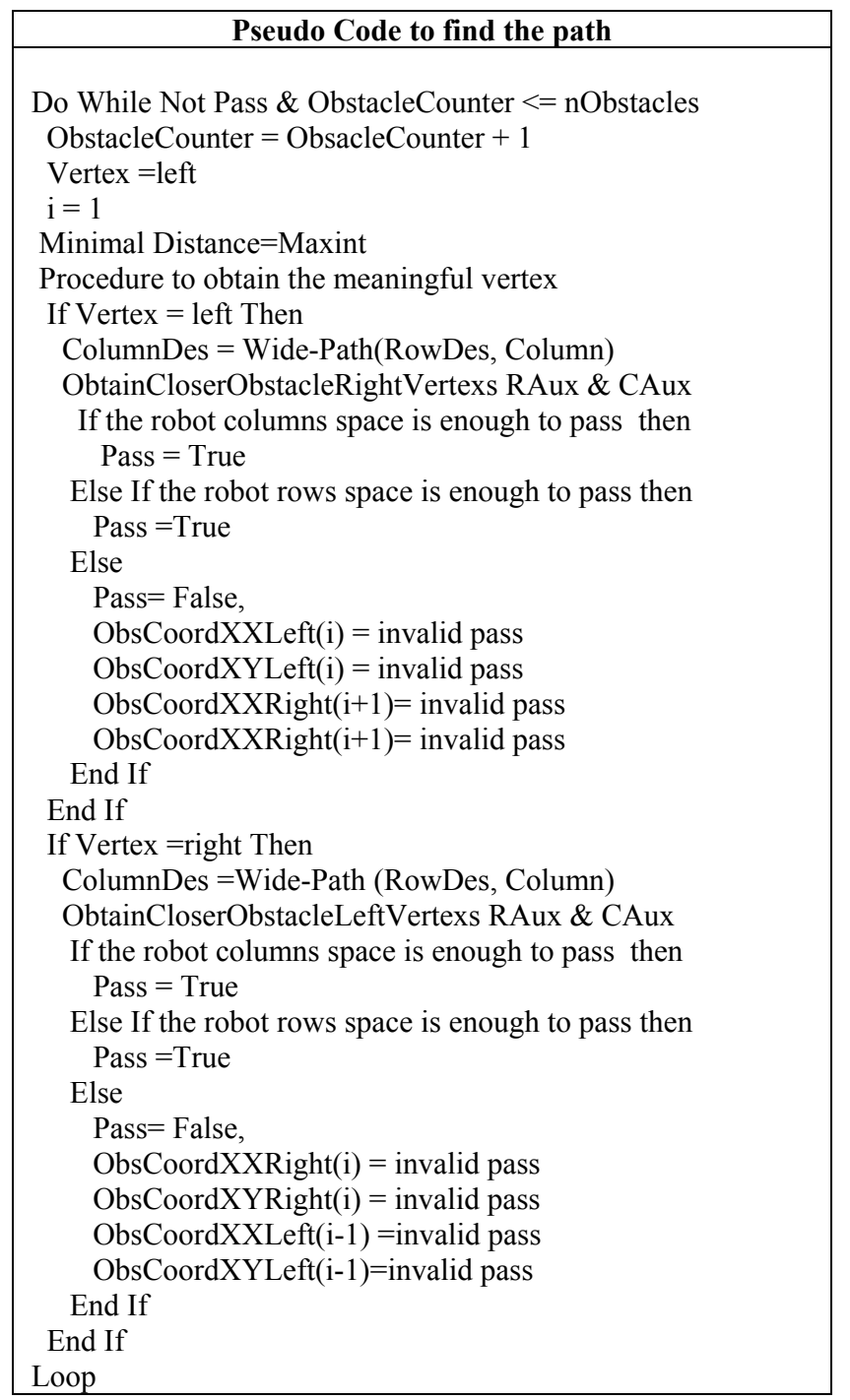

Fig. 4: Once the meaningful vertex is known, a closer path is searched for.

When no path is found the robot should turn, either right or left. One simple rule is to turn based on the better cost function. However, due to the reduced field of view the significant information is only in front of the robot. Therefore, short term, right and left, passed obstacle memory [20] is proposed. The short term memory can be used to avoid repetitive turning actions as well as unsafe trajectories that arise from the narrow field of view.

\section{Results ObTaIned By Using A Mobile Robot With A MONOCUlar PERCEPTION SYSTEM}

In this section the laboratory results obtained using the WMR PRIM [21] are presented. The mechanical structure of the robot PRIM is made of aluminium, with two independent wheels, $16 \mathrm{~cm}$ in diameter, run by two DC motors. The distance between the two wheels is $56.4 \mathrm{~cm}$. A third spherical omni-directional wheel is used to guarantee the stability of the system. The $W P$ radius considered in this work is $35 \mathrm{~cm}$, and the WMR maximal speed is $0.5 \mathrm{~m} / \mathrm{s}$. The maximum continuous torque of each motor is $131 \mathrm{mNm}$. The gear reduction proportion for each motor is 86:1 and thus the total force acting on the robot is near $141 \mathrm{~N}$. Shaft encoders, 500 counts/rev, are placed at the motor axes, which provide 43000 counts for each turn of the wheel. The local perception data are provided by a monocular camera, and are used to plan a feasible trajectory and avoid collisions with obstacles. In the following section we first introduce the machine vision system used to test the methodology performance, and then present different experimental results using the trajectory planning strategy introduced in Section II and the above computer vision method.

\section{A. The Monocular Machine Vision System}

The available scene coordinates appear as an image, in which the camera setup and the pose knowledge are used, and projective perspective is assumed, to make each pixel coordinate correspond to a 3D scene coordinate [22]. Fig. 5 shows the on robot camera configuration considered in this work. $\alpha, \beta$ and $\varphi$ are angles of the vertical and horizontal field of view and the tilt camera pose respectively. The vertical coordinate of the camera is represented by $H$. Using trigonometric relationships, the flat floor scene coordinates can be computed as follows:

$$
\begin{gathered}
Y_{j}=H \tan (\varphi-\alpha / 2+\Delta \alpha) \\
\Delta \alpha=K_{j} \frac{\alpha}{R} \quad\left(0 \leq \mathrm{K}_{\mathrm{j}} \leq R\right) \\
X_{i, j}= \pm \frac{H}{\cos (\varphi-\alpha / 2+\Delta \alpha)} \tan (\Delta \beta) \\
\Delta \beta=K_{i} \frac{\beta}{C} \quad\left(0 \leq \mathrm{K}_{\mathrm{i}} \leq C / 2\right)
\end{gathered}
$$

$K_{i}$ and $K_{\mathrm{j}}$ are parameters used for covering the image pixel discrete space. Thus, $R$ and $C$ represent the image resolution through the total number of rows and columns. It should be noted that for each row position corresponding to scene coordinates $Y_{j}$, there are $C$ column coordinates $X_{i, j}$. The above equations provide the available local map coordinates when no obstacle is detected. Fig. 6 shows a local map provided by the camera, which corresponds to a field of view with a horizontal angle of $48^{\circ}$, a vertical angle of $37^{\circ}$, $H$ set to $109 \mathrm{~cm}$ and a tilt angle of $32^{\circ}$.

The low resolution scene grids are used to speed up the computing process. Hence, image processing methods, such as pyramidal resolution, or down sampling methods can be used to compress images [23].
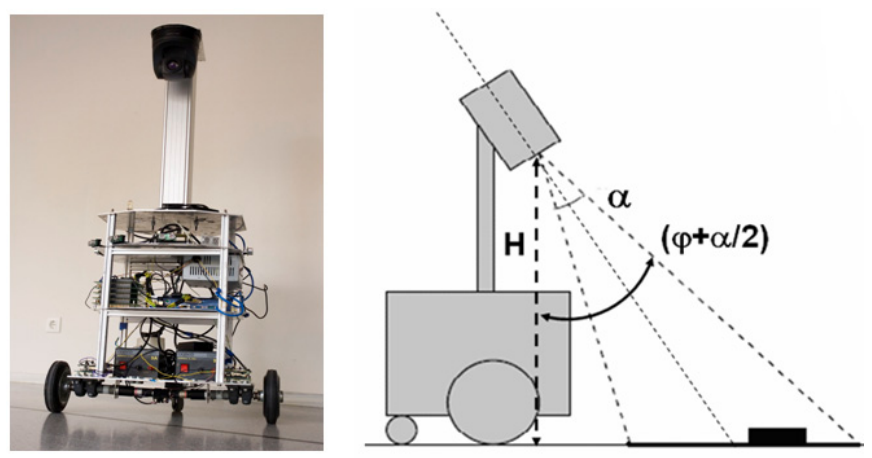

Fig. 5: The robot PRIM I, and the on-robot monocular camera configuration. 


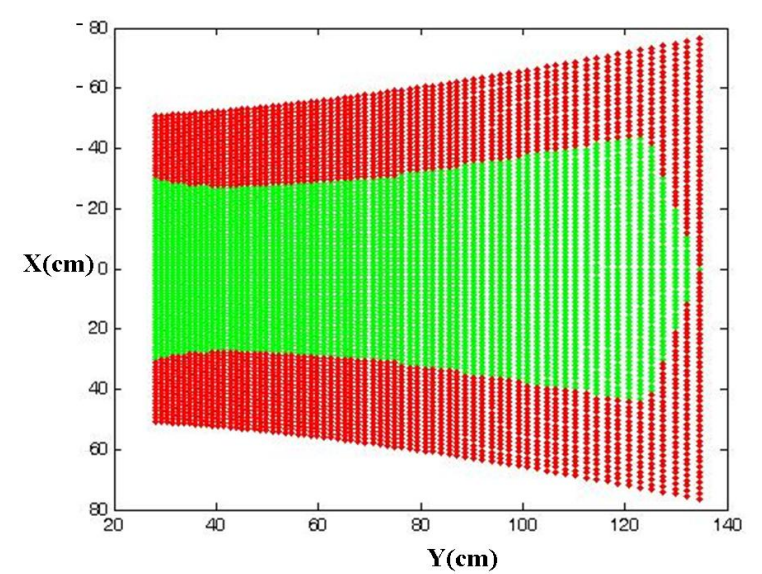

Fig. 6: Local grid coordinates relative to the robot position, with a $96 \times 72$ resolution grid, when no obstacles are detected. The available local cell coordinates are shown in green. The unable cells are drawn in red, due to the physical robot WP.

The results of coordinate maps can be improved by using calibration techniques that allow removing, for instance, the radial distortion [24]. Computer vision research, developed to obtain scene information, has solved many interesting problems using different methods based on an understanding of biological systems. Hence, machine vision systems have used some features of eyes, such as stereopsis, optical flow or accommodation, as meaningful clues [22, 25 and 26]. SVS (Stereo Vision Systems), OFM (Optical Flow Methods) and DFF (Depth from Focus) are all methods that permit 3D scene recovery. Studies comparing SVS and DFF are reported in [27]. The results show that while SVS has greater resolution and sensitivity, DFF has better robustness, requires less computational effort and can deal properly with correspondence and occlusion problems. However, the need for several images of the same scene, acquired with different optical setups, may be considered a significant drawback to using DFF methods in major WMR applications. Using DFF in mobile robotics has been reported in [26], where Noubakhsh used three cameras with almost the same scene to achieve robust and efficient detection. In this work the performance of DFF methods applied to WMRs is analysed using only one frame [28]. By using one image, obstacle positions can be achieved when homogeneous radiance and flat floor constraints are present. Thus, one bit depth can be obtained using the DFF methodology as well as a set of multi-resolution focus thresholds. Complementary information concerning the algorithms implemented and some experimental results can be found in [28].

\section{B. Trajectory Tracking Experimental Results}

The experimental results reported in this section were obtained by using monocular acquired frames, commanding a local coordinate objective $\left(X_{d}, Y_{d}\right)$. Once free and occupied cells were known, the potential field cost function formulated in (1) and (2.a) and the local grid path finder algorithms described in Section II were applied. Note that due to some false positive detection problems the robot is positioned from local desired point to local desired point and then the frame is acquired. Thus, frames containing false positives can be discarded and the path planning strategy can be successfully tested. Hence, different experimental results are reported.

Fig. 7 shows the experiment reported. It is representative of the method's performance and drawbacks; it has been used hallways around the lab. It is shown that when the local objective and potential field is set to $G_{2},(-280,225)$, the local minimum may occur, and a contour tracking policy or an intermediate local objective $G_{l}(455,545)$ will be necessary. Thus when $G_{1}$ is commanded before $G_{2}$ the objective is accomplished. Another aspect to be noted is that in this experiment the constraint wall appearing upside from the trajectory, frames (f) and (g) in Fig. 7, has been modelled using lines placed on the floor that in fact act as obstacles. The experimented tracking behaviour using Euclidean distance as a potential field has shown a dominant right turning behaviour. Therefore, a trajectory from $S$ to $G_{l}$, was tested using (2-b) as a potential field (see Fig. 8) and a smoother trajectory is reported as the WMR is more sensitive to both approaching directions $(X, Y)$.

Finally, the problem of wheel slippage should be mentioned. However, in this work the final error reported, by using the Euclidean distance, was less than $50 \mathrm{~cm}$ in the three experiments shown.

\section{CONCLUSIONS AND FURTHER WORK}

This work shows the implementation of a perception system used as an information source to plan feasible trajectories that bring the robot to a final desired configuration using an attraction potential field. The practical approach has been developed using a monocular perception system; however, the methodology presented can be used with other ranges of sensor systems. Potential field attraction points have shown their effectiveness dealing with reactive behaviours and also with indoor trajectories. Hence, satisfactory results can be obtained with trajectories of nearly $20 \mathrm{~m}$ or even longer.

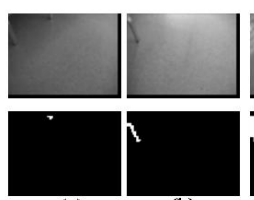

(a)

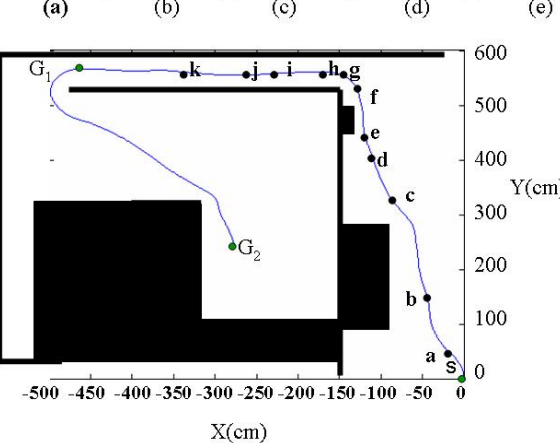

$\mathrm{X}(\mathrm{cm})$

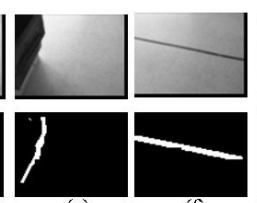

(f)

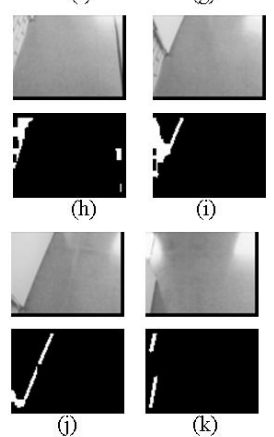

Fig. 7: Trajectory followed from $S$ to $G_{1}$ and significant obstacle frames. The global robot coordinates are in $\mathrm{cm}$ and degrees. $X, Y, \theta$, from where the frames were acquired, are:

(a) $\left(-15,44,129^{\circ}\right)$;(b) $\left(-45,152,99^{\circ}\right)$; (c) $\left(-86,-325,117^{\circ}\right)$;

(d) $\left(-110,402,101^{\circ}\right)$; (e) $\left(-119,441,102^{\circ}\right)$; (f) $\left(-127,524,96^{\circ}\right)$;

(g) $\left(-144,553,96^{\circ}\right)$; (h) $\left(-166,560,178^{\circ}\right)$; (i) $\left(-218,559,181^{\circ}\right)$;

(j) $\left(-257,555,184^{\circ}\right)$; (k) $\left(-346,562,169^{\circ}\right)$. 


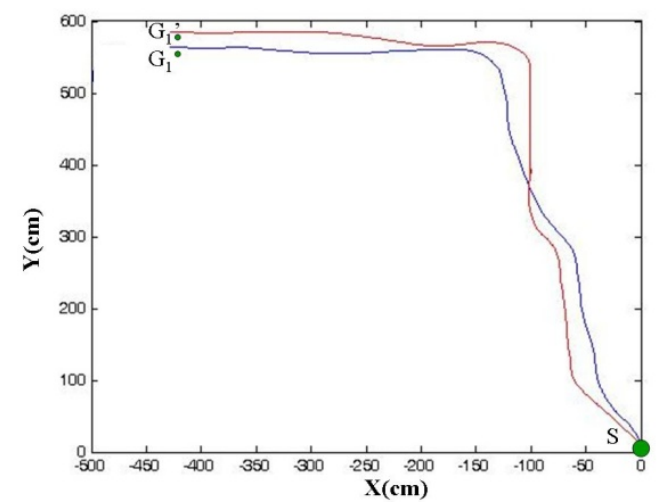

Fig. 8: Trajectory followed from $S$ to $G_{l}$ by using the Euclidean distance as potential field (in blue) or the coordinate magnitude difference (in red).

Radial potential fields based on the Euclidean Distance and coordinate magnitude differences have been proposed. The magnitude differences are lineal and can be understood as a superposition of two potential fields in $X$ and $Y$ directions. Therefore, in some cases smoother behaviour can be expected. The potential field formulation when obstacles are found is reduced to searching for an optimal approach vertex that acts as a local potential field to plan the trajectory. The algorithms used to search for the optimal vertex and feasible obstacle path have also been reported. Hence, only WP is computed for the significant vertexes using Bayesian probabilistic tessellation and the necessary processing time is consequently reduced and computing the complete $\mathrm{C}$ Space is therefore avoided. The present work can be understood as a flexible and practical trajectory planning tool when a local perception occupancy grid is provided. Thus, knowledge of a narrow local grid in which only a few second trajectories can be planned should be enough to plan flexible and accurate global trajectories. Future work should consider different aspects. More experiments need to be carried out on attraction field capabilities and low level control performance. Wheel slippage is a significant drawback to be considered. Thus, natural landmark recognition, of walls, columns or doors, is an important issue to improve the system's performance by setting zero accumulative errors. False obstacle detection, mainly caused by light reflections or changes in the floor radiance homogeneity is another problem to be solved.

\section{ACKNOWLEDGEMENTS}

This work has been partially funded by the Commission of Science and Technology of Spain (CICYT) through the coordinated project DPI-2007-66796-C03-02, the MOMARNE EU project MRTN-CT-2004-505026, the Spanish Ministry of Education and Science under grant CTM2007-64751, and by the Government of Catalonia through the project Xartap -Estació de Muntatge Universal and the consolidated research group's grant SGR200501008 .

\section{REFERENCES}

[1] R. J. Schilling, “Fundamental of Robotics”, Prentice-Hall Inter., 1990.

[2] O. Khatib, "Real time obstacle avoidance for manipulations and mobile robots", Inter. Journal of Robotics Research, vol. 5, pp. 90-98, 1986.
[3] E. Rimon and D. Koditschek, "Exact robot navigation using artificial potential functions, “ IEEE Trans. Robot Autom., vol. 8, no. 5, pp. 501518, Oct. 1992

[4] J. Barraquand, L. Kavraki, J. C. Latombe, T. Y. Li, R. Motwani and P. Raghavan, "A random sampling scheme for path planning", Inter. Journal of Robotics Research, vol. 16, pp. 759-744, 1997.

[5] L. E. Kavraki, P. Svestka, J. C. Latombe and M. H. Overmars, "Probabilistic roadmaps for path planning in high-dimensional configuration spaces", Proc. IEEE Trans. Robotics and Autom., vol. 12, no. 4, pp. 566-580, 1996.

[6] R. Katz and S. Hutchinson, "Efficiently PRMs with Passage Potentials", Proc. ICRA. pp. 889-894, 2006.

[7] R. Geraerts and M. Overmars, "The Corridor Map Method: Real-Time High-Quality Path Planning", Proc. IEEE Inter. Conf. on Robotics and Autom. pp. 1023-1028, 2007.

[8] D. Fox, W. Burgard and S. Thun, "The dynamic window approach to collision avoidance', IEEE Robot. Autom. Mag. Vol. 4, pp. 23-33, Mar. 1997.

[9] P. Ögren and N. Leonard, "A convergent dynamic window approach to obstacle avoidance”, IEEE Trans. Robotics, Vol. 21, no 2, April 2005.

[10]A. Elfes, "Using occupancy grids for mobile robot perception and navigation”, IEEE Computer Society, Vol. 22, pp. 46-57, 1989.

[11]S. Thrun, "Robotic mapping: a survey", Exploring Artificial Intell. in the New Millenium, Morgan Kaufmann, San Mateo, 2002.

[12]C. Coué, C. Pradalier, C. Laugier, T. Fraichard and P. Bessière, "Bayesian Occupancy Filtering for Multitarget Tracking: An Automotive Application”, The Inter. Journal of Robotic Research, 2006.

[13]R. Benenson, S., Petti, T. Fraichard and M. Parent, "Integrating Perception and Planning for Autonomous Navigation of Urban Vehicles", Proc. IEEE Inter. Conf. on Robots and Syst., 2006.

[14]L. Pacheco and N. Luo, "Trajectory Planning with Control Horizon Based on Narrow Local Occupancy Grid”, K. Kozlowky (Ed.), Robot Motion and Control, Springer-Verlag, 2007.

[15] Z. Butler, "Corridor planning for natural agents", Proc. IEEE Inter. Conf. on Robotics and Autom., pp. 499-504, 2006.

[16] C. Coué, Th. Fraichard, P. Bessière and E. Mazer, "Multi Sensor Data Fusion Using Bayesian Programming: an Automative Application", Proc. IEEE Inter. Conf. Intell. Robots and Systems, pp. 442-447, 2002.

[17] C. Fulgenzi, A. Spalanzani and C. Laugier, "Dynamic Obstacle Avoidance in uncertain environment combining PVOs and Occupancy Grid”, Proc. IEEE Inter. Conf. on Robotics and Autom., pp. 16101616, 2007.

[18] S. Quinlan and O. Khatib, "Elastic Bands: Connecting Path Planning and Control", Proc. IEEE Inter. Conf. on Robotics and Autom., pp. 802-807, 1993

[19] L. C. A. Pimienta, G. A. S. Pereira and R. C. Mesquita, "Fully continuous vector fields for mobile robot navigation of discrete triangular regions", Proc. IEEE Inter Conf. on Robotics and Autom., pp. 1992-1997, 2007.

[20] H. Schäfer, M. Proetzsch and K. Berns, "Obstacle Detection in Mobile Outdoor Robots", Proc. Inter. Conf. on Informatics in Control, Autom. and Robotics, pp. 141-148, 2007.

[21] L. Pacheco and N. Luo, "Mobile Robot Local Predictive Control Using a Visual Perception Horizon", Int. Journal of Factory Autom. Robotics and Soft Comp., I. 2, pp. 73-81, April 2007.

[22] B. K. P. Horn, "Robot Vision", McGraw-Hill Book Company, MIT Press Edition, $12^{\text {th }}$ printing 1998.

[23]R. C. Gonzalez and R. E. Woods, “Digital Image Processing,” Prentice Hall Int. Ed., $2^{\text {th }}$ Ed., 2002.

[24] E. Elsayed Hemayed, “A Survey of Camera Self-Calibration,” IEEE Conference on Adv. Video and Signal Based Surv., 2003.

[25] J. Campbell, R. Sukthankar and I. Noubakhsh "Techniques for Evaluating Optical Flow in Extreme terrain," IEEE Int. Conf. Intell. Robots and Systems, 2004.

[26] I. Nourbakhsh, D. Andre, C. Tomasi, and M. R. Genesereth, "Mobile Robot Obstacle Avoidance Via Depth From Focus", Robotics and Autom. Systems, Vol. 22, pp. 151-58, June 1997.

[27] Y. Schechner and N. Kiryati, "Depth from Defocus vs. Stereo: How Different Really Are They?", Proc. IEEE Int. Conf. on Pattern Recognition, vol. 2, 1998.

[28] L Pacheco, X. Cufí, and J. Cobos, "Constrained Monocular Obstacle Perception with Just One Frame”, Lecture Notes in Computer Science, Springer-Verlag, Pattern Recognition and Image Analysis, J. Marti et al. (Eds), pp. 611-619, 2007. 